Supporting Materials for

\title{
Plasmon-Assisted Local Temperature Control to Pattern Individual Semiconductor Nanowires and Carbon Nanotubes
}

Linyou Cao, David N. Barsic, Alex R. Guichard, Mark L. Brongersma*

Department of Materials Science and Engineering, Geballe Laboratory for Advanced Materials, Stanford University, Stanford, CA 94305, USA

*To whom correspondence should be addressed. Email: brongersma@stanford.edu

This PDF file includes:

Fig. S1-S2 


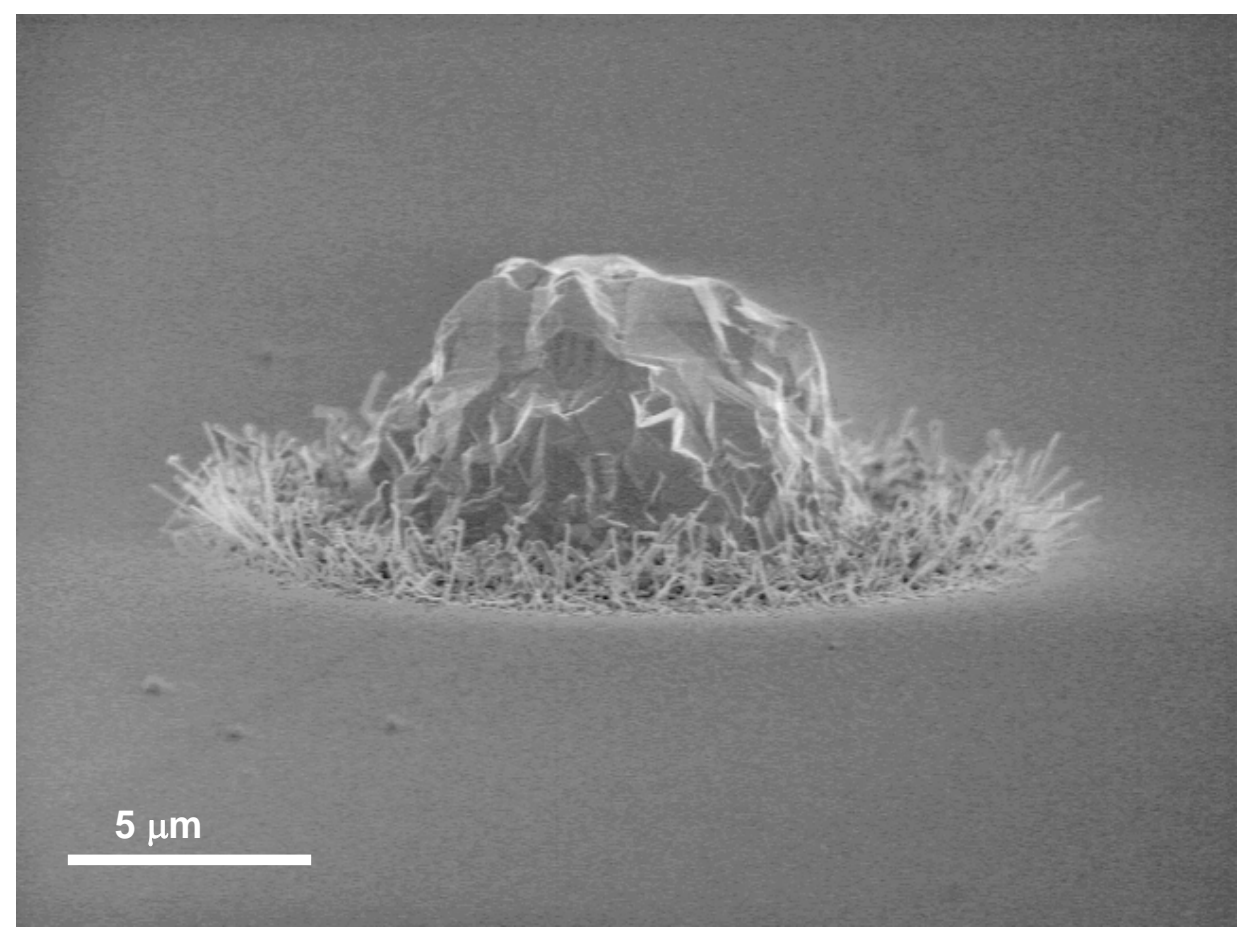

Fig. S1. Scanning electron microscopy image of a large silicon pillar surrounded by band of Si nanowires. This growth morphology was obtained by illuminating a random array of $\mathrm{Au}$ nanoparticles with a $150 \mathrm{~mW}$ Gaussian laser beam focused to a $\sim 18 \mu \mathrm{m}$ radius spot and using silane $\left(\mathrm{SiH}_{4}\right)$ as the precursor gas (partial pressure of 1.6 Torr). In the hot central part of the illuminated region rampant Si growth occurred. At these high power densities the growth is uncontrolled and sometimes more than one pillar is found in the central region, as shown in Fig. 4b in the main text. In a colder band around the central area the ideal conditions for Si nanowire growth occurred. In the even cooler region outside the Si nanowire band the temperatures were too low to induce Si nanowire growth. 


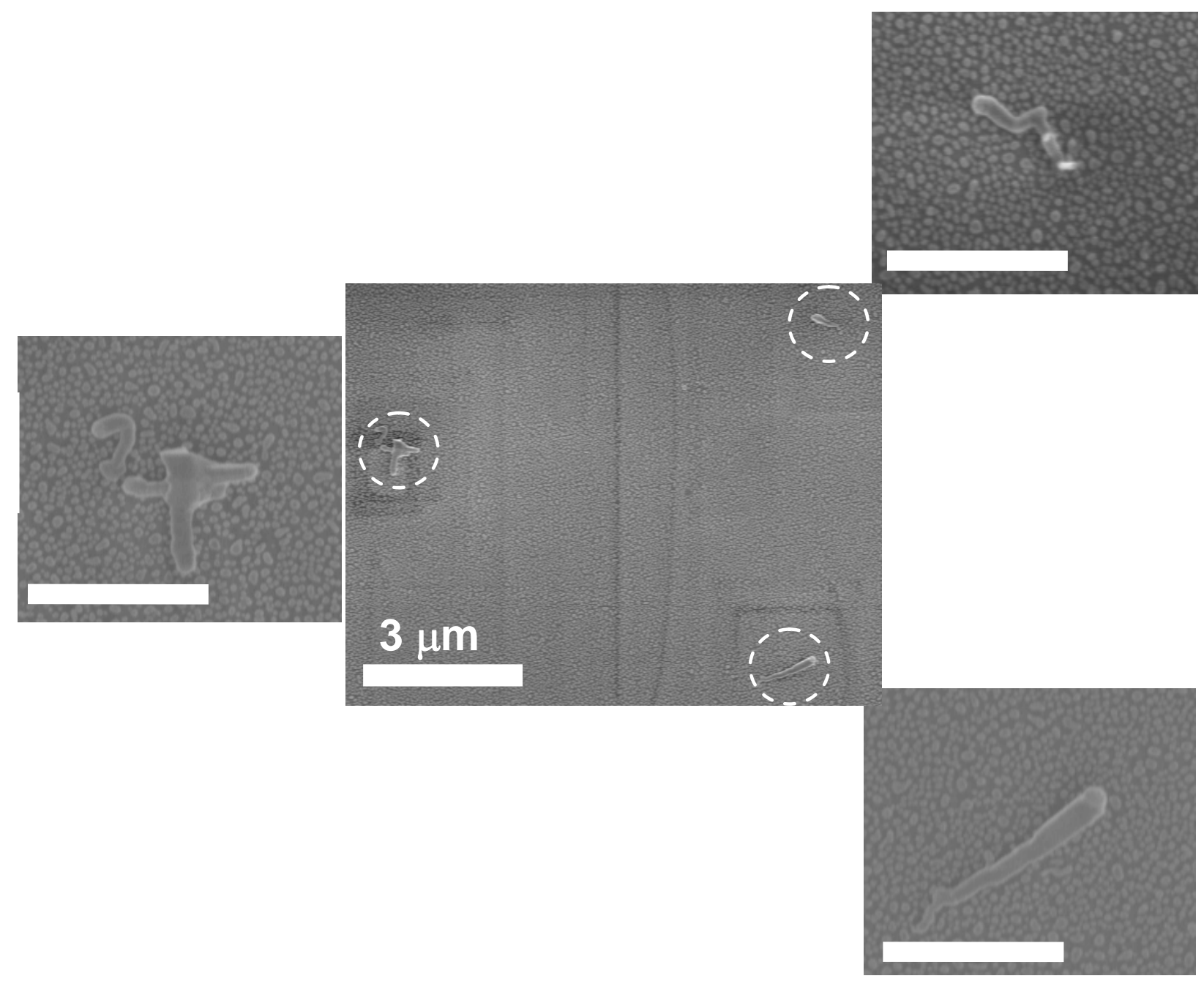

Fig. S2. Three magnified scanning electron microscopy (SEM) images of the Si nanowires shown in Fig. $1 \mathrm{~h}$ of the main paper. The scale bars in the three magnified images are $1 \mu \mathrm{m}$. For convenience, Fig. $1 \mathrm{~h}$ is replotted in the center. 